\title{
Galaxy Disruption Caught in the Act
}

\author{
A. M. Karick \\ School of Physics, University of Melbourne, Victoria 3010, Australia
}

M. J. Drinkwater, M. J. West

University of Queensland, Queensland 4027, Australia

Department of Physics and Astronomy, University of Hawaii, Hilo, HI 96720, USA

\author{
M. D. Gregg, M. Hilker
}

Department of Physics, University of California, Davis, CA 95616, USA Sternwarte der Universität Bonn, Auf dem Hügel 71, 53121, Germany

\begin{abstract}
Direct evidence of stellar material from galaxy disruption in the intra-cluster medium (ICM) relies on challenging observations of individual stars, planetary nebulae and diffuse optical light. Here we show that the ultra-compact dwarf galaxies (UCDs) we have discovered in the Fornax Cluster are a new and easy-to-measure probe of disruption in the ICM. We present spectroscopic observations supporting the hypothesis that the UCDs are the remnant nuclei of tidally "threshed" dwarf galaxies. Deep optical imaging of the cluster has revealed a 43-kpc long arc of tidal debris, flanking a nucleated dwarf elliptical $(\mathrm{dE}, \mathrm{N})$ cluster member. We may be witnessing galaxy threshing in action.
\end{abstract}

Recently a number of spectacular streams of low surface brightness material have been observed in galaxy clusters - evidence that galaxy disruption is an ongoing and important process in galaxy evolution. Material torn from galaxies is dispersed into the intra-cluster medium, contributing up to $10-20 \%$ of the cluster luminosity.

A small sample of ultra-compact dwarf galaxies were discovered during an all-object Fornax Cluster Spectroscopic Survey (FCSS) using the AngloAustralian Telescope 2dF spectrograph (Drinkwater et al. 1999). These old stellar systems are 10 times more luminous than the brightest globular clusters associated with the central Fornax Cluster galaxy NGC 1399, with intrinsic sizes of $\sim 100$ pc and B-band magnitudes ranging from -13 to -11 (Karick et al. 2003). Truly intergalactic, they are not associated with any bright cluster galaxy. Our most favoured hypothesis for their origin is that they are the remnant nuclei of infalling nucleated dwarf elliptical ( $\mathrm{dE}, \mathrm{Ns}$ ) galaxies which have been tidally "threshed" by large galaxies or by the cluster tidal field (Bekki et al. 2001).

The internal velocity dispersions of the Fornax Cluster UCDs were measured using the VLT and Keck Telescopes (Drinkwater et al. 2003). The velocity dispersions range from $24-37 \mathrm{~km} \mathrm{~s}^{-1}$, considerably higher than those of Galactic 


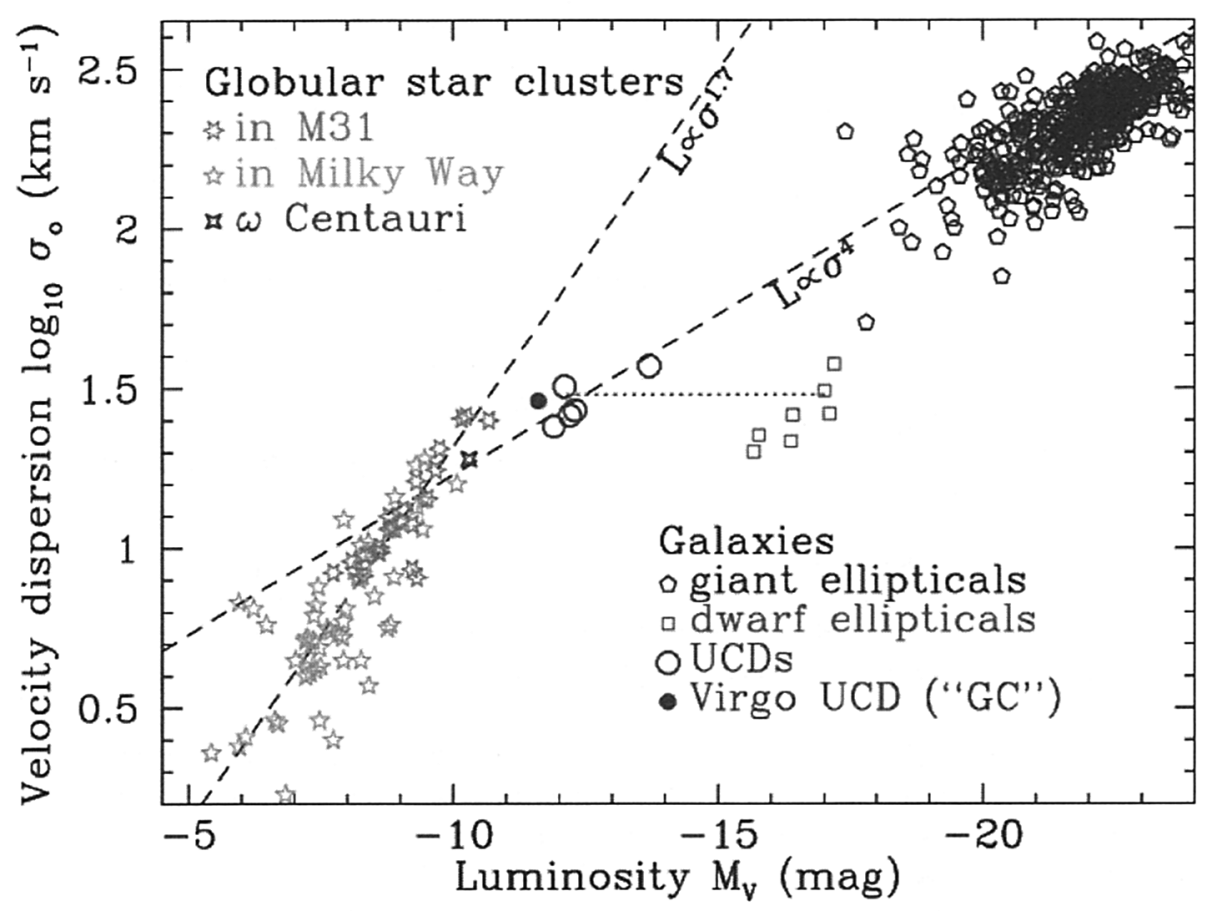

Figure 1. Comparison of the internal dynamics of the UCDs with GCs and normal galaxies. The horizontal line shows the predicted effect of galaxy threshing.

GCs. The UCDs lie well off the globular cluster $\mathrm{L} \propto \sigma^{1.7}$ relation (Fig 1 ) in a previously unoccupied region. The locations of the UCDs and the dE,Ns on the diagram strongly support the galaxy threshing model. A normal dE,N halo, accounting for $\sim 98 \%$ of the $\mathrm{dE}, \mathrm{Ns}$ luminosity, is tidally stripped therefore reducing the total luminosity by a factor of 100 but barely changing the central velocity dispersion. Over time this disruption contributes diffuse stellar material to the ICM. The surviving nuclei are dispersed into intra-cluster space or added to the envelopes of brighter galaxies, where they can masquerade as stars. This process may explain the observed high "specific frequency" of GCs in central cluster galaxies.

We also obtained deep multicolour $(\mathrm{u}, \mathrm{g}, \mathrm{r}, \mathrm{i}, \mathrm{z})$ imaging of the central 2 square degree region of the Fornax Cluster using the CTIO 4m MOSAIC Telescope $\left(0.27^{\prime \prime}\right.$ pix $\left.\approx 27 \mathrm{pc}\right)$. This revealed a $43 \mathrm{kpc}$-long plume of tidal debris 0.5 degrees from the cluster centre, flanking the $\mathrm{dE}, \mathrm{N}$ cluster member FCC 252 (Fig 2). The diffuse stellar material has $\mu_{g}=26.9 \mathrm{mag} \operatorname{arcsec}^{-2}$ and $M_{g} \approx-14$, comparable to faint Local Group dwarf spheroidals (Mateo 1998). The proximity of the debris suggests we may be witnessing galaxy threshing in action. The stellar populations of the UCDs, dE,Ns and GCs associated with NGC 1399 will be determined from two-colour diagrams. This will provide a further test of the 


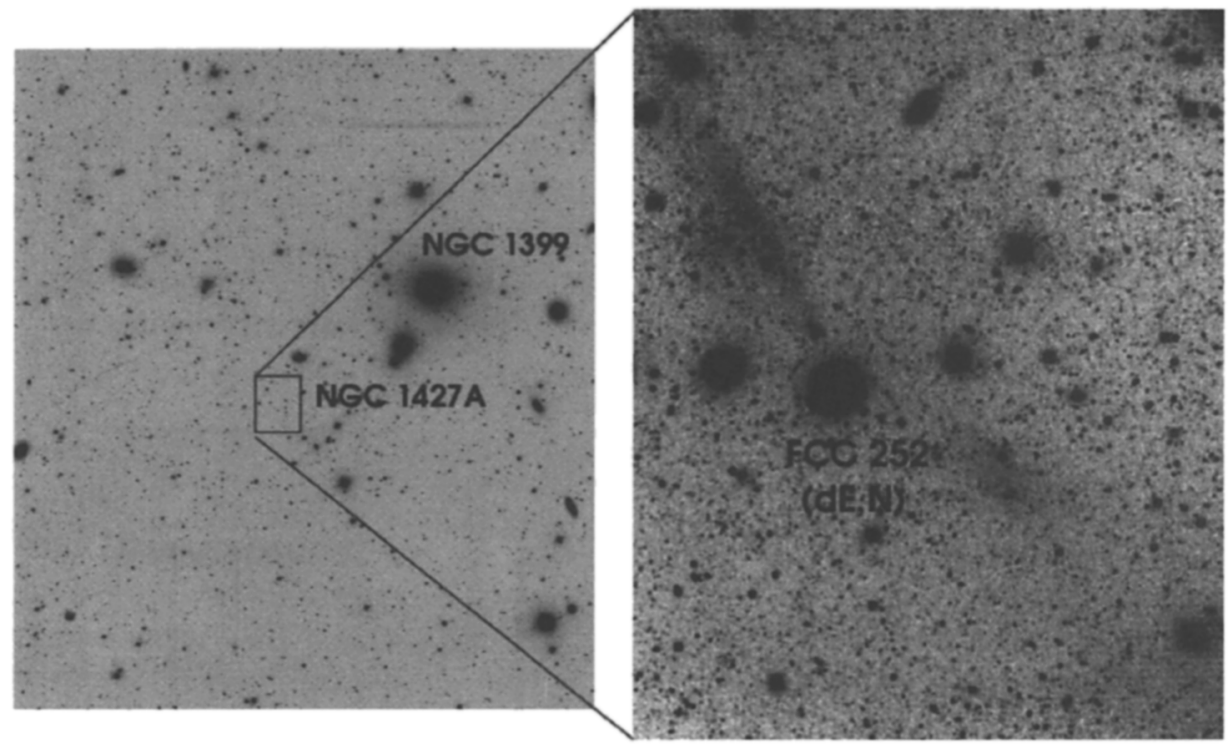

Figure 2. Left: A $0.5^{\circ} \times 0.5^{\circ}$ region of the Fornax Cluster. NGC $1427 \mathrm{~A}$ is part of an infalling subcluster and shows evidence of interaction with the ICM. Right: An $8^{\prime} \times 8^{\prime}$ region showing the curved arc of tidal debris, extending from upper left to lower right through the $\mathrm{dE}, \mathrm{N}$, FCC 252.

galaxy threshing hypothesis. We require higher resolution observations of FCC 252 and the debris to determine their possible tidal relationship.

Acknowledgments. We would like to thank our collaborator R. Jurek.

\section{References}

Bekki et al. 2001, ApJ, 552, L105

Drinkwater et al. 1999, ApJ, 511, L97

Drinkwater et al. 2003, Nature, 423, 519

Karick et al. 2003, MNRAS, 344, 188

Mateo 1998, ARA\&A, 36, 435 